\title{
Optimal Release Policy for Prophylactic Biological Control
}

\author{
Ludovic Mailleret $^{1}$ and Frédéric Grognard ${ }^{2}$ \\ 1 Urih, InRA, BP 167 - 06903 Sophia Antipolis Cedex, France \\ ludovic.mailleret@antibes.inra.fr \\ 2 Comore, Inria, BP 93 - 06902 Sophia Antipolis Cedex, France \\ frederic.grognard@sophia.inria.fr
}

Summary. In this paper, we study the prophylactic biological control strategy for greenhouse crops protection. The method consists in the preventive installation of natural enemies to fight against an invading pest, using discrete augmentative (i.e. inondative) releases of the natural enemies. We consider a simple non negative prey (pest) - predator (natural enemy) model in ordinary differential equations together with discrete augmentation of the predator population at constant frequency. Assuming we have a fixed budget to spend in natural enemies releases per time unit, we show the stability and efficiency of the prophylactic biological control strategy (i.e. the pest is eradicated) if this budget is larger than some value. Then we show that the optimal strategy to minimize worst case damage is to use the most frequent (and thus smallest) releases.

\section{Introduction}

Due to the high costs induced by greenhouse crops production, a particular attention must be given to reduce to the lowest the damage caused by pests. Within this context, prophylatic methods (i.e. prevention), able to fight pests at the time of their introduction (not after some delay) are more effective than curative ones. Till now, these methods are most widely used for spraying of chemicals; however, due to social (growers and consumers health), environmental (water pollution) and ecological (development of resistance by pests) considerations, chemical pesticides should be used with great care [3]. An alternative strategy is to use biological control, i.e. to introduce natural enemies to fight against pests, instead of chemicals.

Assuming that crop damage are low and then that crop is not limiting for pest growth, we consider a prey (pest) - predator (natural enemy) system modeled with two non negative ordinary differential equations. We consider that the crop never ends and study on this horizon the augmentative biological control method: the increase, at a fixed time period and with a fixed number, 
of the predator population. We consider that we have a constant budget (i.e. number of natural enemies) to spend for biological control per time unit.

We show the existence and stability of a periodic pest-eradicated solution (i.e. prophylactic solution) iff the budget is larger than a value determined by the model parameters. Moreover, as the prophylactic solution runs, the natural enemies are able to eradicate any level of unforeseen pest infestation. It is to be noted that this first part uses a method quite similar to the one proposed by [5] in the context of mathematical epidemiology and rephrased by [4] in the context of mathematical modelling of crop protection.

We then suppose that the prophylactic solution runs while an infestation occurs and look for the releasing period that minimizes the worst case (according to the moment of infestation) crop damage due to the pest. We show that most frequent releases is the more efficient strategy with respect to uncertainties on the invading pest population level and on the model parameters.

\section{Model Description and Analysis}

\subsection{Model Description}

Since we consider the crop as non-limiting for pest growth, we simplify the tri-trophic ecosystem "crop - pest - natural enemies" as a bi-trophic one with only a prey (pest) and a predator population (natural enemies). According to classical population dynamics, we have the following non negative model:

$$
\left\{\begin{array}{l}
\dot{x}=f(x)-g(x) y \\
\dot{y}=k g(x) y-m y
\end{array}\right.
$$

$x$ denoting the preys and $y$ the predators. $f(x)$ denotes the growth speed of the preys, $g(x)$ the predation rate, $k$ the yield associated with the predation for the predators and $m$ the mortality rate of the predators. Since biological processes are always difficult to model, we only assume weak qualitative hypotheses on the (smooth enough) functions $f($.$) and g($.$) :$

\section{Hypotheses 1}

- $f(0)=0, f^{\prime}(0)>0$ and $\forall x \geq 0, f^{\prime \prime}(x) \leq 0$

- $g(0)=0, g^{\prime}(0)>0$ and $\forall x \geq 0, g^{\prime \prime}(x) \geq 0$

We now model the periodic release of predators; let us suppose that we have a fixed budget (i.e. fixed number of natural enemies) per time unit to spend for crop protection with prophylactic biological control. Let $\mu$ be the number of predators that can be released per time unit, then at each time period $T, \mu T$ predators are added to the predator population $y$; we have the following non negative system of impulsive differential equations [1]:

$$
\left\{\begin{array}{l}
\dot{x}=f(x)-g(x) y \\
\dot{y}=k g(x) y-m y \\
\forall n \in \mathbb{N}, y\left(n T^{+}\right)=y(n T)+\mu T
\end{array}\right.
$$




\subsection{Model Analysis}

Theorem 1. Under Hypotheses 1, model (1) possesses a prophylactic periodic solution (corresponding to the pest eradication):

$$
\left(x_{p}(t), y_{p}(t)\right)=\left(0, \frac{\left.\mu T e^{-m(t} \bmod T\right)}{1-e^{-m T}}\right)
$$

which is (locally) stable iff:

$$
\mu>\frac{m f^{\prime}(0)}{g^{\prime}(0)}
$$

Moreover, as the prophylactic solution runs and (3) holds, the natural enemies are able to eradicate any level of unforeseen pest infestation and there exists a release period $T_{\min }$ under which the pest population is always decreasing.

Proof. We first focus on the existence and stability of the prophylactic solution i.e. on the dynamics of $y(t)$ as no prey is present; we get:

$$
\left\{\begin{array}{l}
\dot{x}=0 \\
\dot{y}=-m y \\
\forall n \in \mathbb{N}, y\left(n T^{+}\right)=y(n T)+\mu T
\end{array}\right.
$$

that yields: $y\left((n+1) T^{+}\right)=y\left(n T^{+}\right) e^{-m T}+\mu T$. It is clear that the sequence $\left(y\left(n T^{+}\right)\right)_{n \in \mathbb{N}}$ has a single and globally stable equilibrium $y^{\star}=\mu T /\left(1-e^{-m T}\right)$. Then, system (4) possesses the single globally stable periodic solution (2).

We now prove the (local) stability of the prophylactic solution for system (1), that is to say with the presence of a prey population. We must then consider small perturbations around the periodic solution $\left(x_{p}(t), y_{p}(t)\right)$, i.e. we consider $(x(t), y(t))=\left(x_{p}(t), y_{p}(t)\right)+(\tilde{x}(t), \tilde{y}(t))$. We get:

$$
\left\{\begin{array}{l}
\dot{\tilde{x}}=f(\tilde{x})-g(\tilde{x})\left(\tilde{y}+y_{p}(t)\right) \\
\dot{\tilde{y}}=k g(\tilde{x})\left(y_{p}(t)+\tilde{y}\right)-m \tilde{y}
\end{array}\right.
$$

At first order in $\tilde{x}$ and $\tilde{y}$, we have:

$$
\left\{\begin{array}{l}
\dot{\tilde{x}}=\left(f^{\prime}(0)-g^{\prime}(0) y_{p}(t)\right) \tilde{x} \\
\dot{\tilde{y}}=k g^{\prime}(0) y_{p}(t) \tilde{x}-m \tilde{y}
\end{array}\right.
$$

which is a linear system (in $\tilde{x}$ and $\tilde{y}$ ) with periodic coefficients. We then use Floquet's theory which ensures the local stability of $(0,0)$ for system $(6)$ (and thus of $\left(x_{p}(t), y_{p}(t)\right)$ for (1)) iff the Floquet multipliers of the system have their absolute values lower than one (see e.g. [2] for the theory). In our case, the two Floquet multipliers are $e^{-m T} \in(0,1)$ and $e^{\int_{0}^{T}\left(f^{\prime}(0)-g^{\prime}(0) y_{p}(\tau)\right) d \tau}>0$ that is lower than one iff $\mu>m f^{\prime}(0) / g^{\prime}(0)$. Then $\left(x_{p}(t), y_{p}(t)\right)$ is locally stable iff (3) holds.

We now come back to system (5). From Hypotheses 1 and since $y_{p}(t) \geq 0$, system (5) is non-negative. We now consider a forward trajectory of system 
(5) initiated at time $t_{0} \geq 0$ at $\left(\tilde{x}_{0}, 0\right) \geq 0$. From $\tilde{y} \geq 0$ and Hypotheses 1 , we have:

$$
\dot{\tilde{x}} \leq\left(f^{\prime}(0)-g^{\prime}(0) y_{p}(t)\right) \tilde{x}
$$

Thus, since $y_{p}(t)$ is $T$-periodic:

$\tilde{x}(t) \leq x_{0} e^{\int_{t_{0}}^{l T}\left(f^{\prime}(0)-g^{\prime}(0) y_{p}(\tau)\right) d \tau} e^{(k-l) \int_{0}^{T}\left(f^{\prime}(0)-g^{\prime}(0) y_{p}(\tau)\right) d \tau} e^{\int_{k T}^{t}\left(f^{\prime}(0)-g^{\prime}(0) y_{p}(\tau)\right) d \tau}$

with $k$ the integer part of $t / T$ and $l$ the integer part of $t_{0} / T$ plus one. Suppose (3) holds, then $\int_{0}^{T}\left(f^{\prime}(0)-g^{\prime}(0) y_{p}(\tau)\right) d \tau<0$. Since $k$ goes to infinity as $t$ does, $\tilde{x}(t)$ converges asymptotically to 0 .

We come back to equation (7), we clearly have:

$$
\dot{\tilde{x}} \leq\left(f^{\prime}(0)-g^{\prime}(0) \min _{t} y_{p}(t)\right) \tilde{x}
$$

$y_{p}$ reaches its minimum at $t=T^{-} \bmod T$ and is equal to $\mu T /\left(e^{m T}-1\right)$ which is a decreasing function of $T$ on $(0,+\infty)$ from $+\infty$ to 0 . Let us define $T_{\text {min }}>0$ such that $\min y_{p}\left(T_{\text {min }}\right)=f^{\prime}(0) / g^{\prime}(0)$; then $\forall T<T_{\text {min }}$, the right hand side of (8) is negative and $\tilde{x}(t)$ is a decreasing function of $t$.

\section{Optimization of the Release Policy}

\subsection{Statement of the Problem}

From now on, we consider that the prophylactic solution (2) runs as a pest population $x_{0}$ invades the crop at a time $t_{0} \in[0, T$ ) (without loss of generality). We consider that the invading population $x_{0}$ is small so that the linear system (6) is a good approximation of system (1). Moreover we assume that we have chosen $T$ so that the pest population is always decreasing i.e. $T \in\left(0, T_{\min }\right)$.

To evaluate crop damage due to the pest, we use the concept of "Economic Injury Level" (EIL) that has been introduced from the early bases of theoretical biological control [6]. EIL (denoted $\bar{x}$ in the sequel) is defined as the lowest (positive) pest population level that will cause economic losses on the crop. For a fixed $x_{0}>\bar{x}$, we claim that the lower the time the pest population is above $\bar{x}$ the lower are the crop damage. Then we look for the release period $T<T_{\min }$ that minimizes the time spent by $x$ above $\bar{x}$ (denoted $\Pi$ in the following) for its worst $t_{0}$.

\subsection{Main Result}

We first look for the $t_{0}$ that maximizes the damage time $\Pi$, we have:

Lemma 1. Suppose $x_{0}>\bar{x}$ and $T \in\left(0, T_{\min }\right)$ are fixed, then one of the following holds:

$i$ - $\exists k \in \mathbb{N}, x_{0} \exp \left(\left(f^{\prime}(0)-\frac{\mu g^{\prime}(0)}{m}\right) k T\right)=\bar{x}$ then $\Pi=k T$

$i i-\exists k \in \mathbb{N}, \max _{t_{0}} \Pi\left(t_{0}\right)=\Pi\left(t_{0}^{*}\right)=(k+1) T-t_{0}^{*}$ (i.e. $\left.x((k+1) T)=\bar{x}\right)$ 
We will first show case (i-) and prove that, otherwise, either case (ii-) holds or $\Pi$ is maximum at $t_{0}^{*}=0$, this latter case being impossible.

Proof. Suppose that $\Pi\left(t_{0}\right)$ is maximum for $t_{0}^{*} \in(0, T)$ and such that $\Pi\left(t_{0}^{*}\right) \in$ $(k T,(k+1) T)$ for some integer $k$. Pick $t_{0}^{m}<t_{0}^{*}<t_{0}^{M}$ in $(0, T)$ such that $\Pi\left(t_{0}\right) \in[k T,(k+1) T)$ for all $t_{0} \in\left[t_{0}^{m}, t_{0}^{M}\right]$. Within this set, define $\tau\left(t_{0}\right)$ as:

$$
\Pi\left(t_{0}\right)=k T-t_{0}+\tau\left(t_{0}\right)
$$

Integrating (6) between $t_{0}$ and $t_{0}+\Pi\left(t_{0}\right)$, we get (with $\tau$ standing for $\tau\left(t_{0}\right)$ ):

$$
x\left(t_{0}+\Pi\left(t_{0}\right)\right)=x_{0} e^{f^{\prime}(0)\left(k T-t_{0}+\tau\right)} e^{\left(\frac{g^{\prime}(0) \mu T}{m\left(1-e^{-m T}\right)}\left(-e^{-m t_{0}}+k\left(e^{-m T}-1\right)+e^{-m \tau}\right)\right)}
$$

which is, from the definition of $\Pi$, equal to $\bar{x}$.

To have a maximum of $\Pi$ at $t_{0}^{*}$, we need $\frac{d \Pi}{d t_{0}}\left(t_{0}^{*}\right)=0$. Thus from (9) we must have $\frac{d \tau}{d t_{0}}\left(t_{0}^{*}\right)=1$. We differentiate (10) with respect to $t_{0}$ and get:

$$
\frac{d \tau}{d t_{0}}\left(t_{0}\right)=\frac{\left(\frac{g^{\prime}(0) \mu T}{1-e^{-m T}}\right) e^{-m t_{0}}-f^{\prime}(0)}{\left(\frac{g^{\prime}(0) \mu T}{1-e^{-m T}}\right) e^{-m \tau\left(t_{0}\right)}-f^{\prime}(0)}
$$

Clearly we must have $\tau\left(t_{0}^{*}\right)=t_{0}^{*}$. Thus from (10) there must exist an integer $k$ such that:

$$
x_{0} e^{\left(f^{\prime}(0)-\frac{\mu g^{\prime}(0)}{m}\right) k T}=\bar{x}
$$

and $\Pi=k T$ does not depend on $t_{0}$ (case (i-)). Otherwise $\Pi$ has no extremum at $t_{0}^{*}$ such that $t_{0}^{*} \in(0, T)$ and $\Pi\left(t_{0}^{*}\right) \in(k T,(k+1) T)$.

Two cases remain to be studied, either $\Pi$ is maximum for $t_{0}^{*}=0$ or $t_{0}^{*}$ is such that $t_{0}^{*}+\Pi\left(t_{0}^{*}\right)=(k+1) T ; t_{0}^{*}=T$ or $t_{0}^{*}+\Pi\left(t_{0}^{*}\right)=k T$ might be studied by $k$ reparametrization.

Assume $t_{0}^{*}=0$. Then from (11) and (9) the right derivative of $\Pi$ at $t_{0}^{*}$ is:

$$
\frac{d \Pi}{d t_{0}}\left(t_{0}^{*}=0^{+}\right)=\frac{\left(\frac{g^{\prime}(0) \mu T}{1-e^{-m T}}\right)-f^{\prime}(0)}{\left(\frac{g^{\prime}(0) \mu T}{1-e^{-m T}}\right) e^{-m \tau\left(t_{0}^{*}\right)}-f^{\prime}(0)}-1
$$

Since $T<T_{\min }$, we have from $(8) f^{\prime}(0)<g^{\prime}(0) \mu T e^{-m T} /\left(1-e^{-m T}\right)$. Then both the numerator and denominator are positive, the former being larger than the latter. $\frac{d \Pi}{d t_{0}}\left(t_{0}^{*}=0^{+}\right)$is then positive and $\Pi$ is minimum at $t_{0}=0$.

Assume now that $t_{0}^{*}$ is such that $t_{0}^{*}+\Pi\left(t_{0}^{*}\right)=(k+1) T$. Then from (9) $\tau\left(t_{0}^{*}\right)=T$ and the left derivative of $\Pi$ at $t_{0}^{*}$ is:

$$
\frac{d \Pi}{d t_{0}}\left(t_{0}^{*-}\right)=\frac{\left(\frac{g^{\prime}(0) \mu T}{1-e^{-m T}}\right) e^{-m t_{0}^{*}}-f^{\prime}(0)}{\left(\frac{g^{\prime}(0) \mu T}{1-e^{-m T}}\right) e^{-m T}-f^{\prime}(0)}-1
$$


In a very same way as in the previous case, since $T<T_{M}$ we show that this derivative is positive. Similarly one can show that the right derivative of $\Pi$ at $t_{0}^{*}$ is negative. This can be performed through the reparametrization of $k$ as $k+1$ while noticing that this corresponds to $\tau\left(t_{0}^{*}\right)=0$. Then for the considered $x_{0}$ and $T, \Pi$ is maximum for $t_{0}^{*}$ s.t.: $t_{0}^{*}+\Pi\left(t_{0}^{*}\right)=(k+1) T$.

Lemma 1 is quite natural. Indeed, the decrease that takes place between times $T$ and $k T$ is independent of $t_{0}$. The worst case should then contain the end of the first-time interval where the predators are scarce rather than the beginning of the last interval where they are abundant.

We now focus on the evolution of the maximum of $\Pi$ according to $t_{0}$ as the release period $T$ varies in $\left(0, T_{\text {min }}\right)$.

Theorem 2. Suppose $x_{0}$ is fixed. Let:

$$
T_{1}=\frac{m\left(\ln \left(x_{0}\right)-\ln (\bar{x})\right)}{\mu g^{\prime}(0)-m f^{\prime}(0)}
$$

Then the following hold:

$i$ - there exists a $n_{0} \in \mathbb{N}^{*}$ such that $\min _{T<T_{\text {min }}} \max _{t_{0}} \Pi=T_{1}$ is reached at $T=T_{n}=\frac{T_{1}}{n}$ for all integer $n>n_{0}$.

$i i-\forall n \geq n_{0}, \Delta \Pi_{n}=\left(\max _{T \in\left[T_{n+1}, T_{n}\right]} \max _{t_{0}} \Pi-\min _{T} \max _{t_{0}} \Pi\right)$ is a decreasing function of $n$. Moreover it tends to 0 as $n$ tends to infinity.

Proof. Consider $T_{1}$; it is clear that there exists an integer $n_{0}$ such that for all integer $n>n_{0}, T_{n}<T_{\text {min }}$. Now consider a $T_{n}$ with $n>n_{0}$; using (12), we have:

$$
x_{0} e^{\left(f^{\prime}(0)-\frac{\mu g^{\prime}(0)}{m}\right) n T_{n}}=x_{0} e^{\left(f^{\prime}(0)-\frac{\mu g^{\prime}(0)}{m}\right) T_{1}}=\bar{x}
$$

Such a $T_{n}$ corresponds to case (i-) in Lemma 1 , thus $\Pi=T_{1}$.

Now we show that $\Pi=T_{1}$ is the minimum (according to $T$ ) of $\max _{t_{0}} \Pi$. Suppose that we have $T \neq T_{n}\left(\forall n \geq n_{0}\right)$. According to case (ii-) in Lemma 1 , $\exists k \in \mathbb{N}$ such that $\max _{t_{0}} \Pi=(k+1) T-t_{0}^{*}$ and $x((k+1) T)=\bar{x}$. Using $(10)$ we have:

$$
x_{0} e^{f^{\prime}(0)\left((k+1) T-t_{0}^{*}\right)} e^{\frac{g^{\prime}(0) \mu T}{m}\left(\frac{1-e^{-m t_{0}^{*}}}{1-e^{-m T}}+(k+1)\right)}=\bar{x}
$$

Which, with a little effort, yields:

$$
(k+1) T-t_{0}^{*}=T_{1}+\frac{\mu g^{\prime}(0)}{\mu g^{\prime}(0)-m f^{\prime}(0)}\left(\frac{1-e^{-m t_{0}^{*}}}{1-e^{-m T}} T-t_{0}^{*}\right)
$$

From $(3), \mu g^{\prime}(0)-m f^{\prime}(0)>0$ and since $t_{0}^{*}<T$ it can easily be shown that:

$$
\left(\frac{1-e^{-m t_{0}^{*}}}{1-e^{-m T}} T-t_{0}^{*}\right) \geq 0
$$

Then, $(k+1) T-t_{0}^{*}=\max _{t_{0}} \Pi$ is larger than $T_{1}$ which gives part (i-) of Theorem 2 . 
Now we prove part (ii-). From (i-) and (13) we have for the corresponding $t_{0}^{*} \in\left[0, T_{n}\right]:$

$$
\begin{aligned}
\Delta \Pi_{n} & =\max _{T \in\left[T_{n+1}, T_{n}\right]} \max _{t_{0}} \Pi-\min _{T} \max _{t_{0}} \Pi \\
& =\frac{\mu g^{\prime}(0)}{\mu g^{\prime}(0)-m f^{\prime}(0)} \max _{T \in\left[T_{n+1}, T_{n}\right]}\left(\frac{1-e^{-m t_{0}^{*}}}{1-e^{-m T}} T-t_{0}^{*}\right)
\end{aligned}
$$

We now look for disjoint sets, depending on $n$, that contain $\Delta \Pi_{n}$.

We first notice that $\frac{1-e^{-m t_{0}^{*}}}{1-e^{-m T}} T$ belongs to $\left[\frac{1-e^{-m t_{0}^{*}}}{1-e^{-m T_{n+1}}} T_{n+1}, \frac{1-e^{-m t_{0}^{*}}}{1-e^{-m T_{n}}} T_{n}\right]$ for all $T \in\left[T_{n+1}, T_{n}\right]$ and with $t_{0}^{*}$, defined by case (ii-) of Lemma 1 for $k=n$, depending on $T$. Since $(n+1) T-t_{0}^{*}(T)=T_{1}$ (case (i-)) at $T=T_{n+1}$ and $T=T_{n}$, we have $t_{0}^{*}\left(T_{n+1}\right)=0$ and $t_{0}^{*}\left(T_{n}\right)=T_{n}$. Then, for all $s \in\left[0, T_{n}\right], \exists T \in$ $\left[T_{n+1}, T_{n}\right]$ such that $t_{0}^{*}(T)=s$. Thus:

$$
\begin{aligned}
\max _{T \in\left[T_{n+1}, T_{n}\right]}\left(\frac{1-e^{-m t_{0}^{*}}}{1-e^{-m T}} T-t_{0}^{*}\right) & \leq \max _{T \in\left[T_{n+1}, T_{n}\right]}\left(\frac{1-e^{-m t_{0}^{*}}}{1-e^{-m T_{n}}} T_{n}-t_{0}^{*}\right) \\
& =\max _{t_{0}^{*} \in\left[0, T_{n}\right]}\left(\frac{1-e^{-m t_{0}^{*}}}{1-e^{-m T_{n}}} T_{n}-t_{0}^{*}\right)
\end{aligned}
$$

Differentiating $\left(\frac{1-e^{-m t_{0}^{*}}}{1-e^{-m T_{n}}} T_{n}-t_{0}^{*}\right)$ with respect to $t_{0}^{*}$, we show that it reaches its maximum for:

$$
t_{0}^{*}=\frac{1}{m} \ln \left(\frac{m T_{n}}{1-e^{-m T_{n}}}\right)
$$

Then:

$$
\begin{aligned}
\max _{T \in\left[T_{n+1}, T_{n}\right]}\left(\frac{1-e^{-m t_{0}^{*}}}{1-e^{-m T}} T-t_{0}^{*}\right) & \leq \frac{1}{m}\left(\frac{m T_{n}}{1-e^{-m T_{n}}}-1-\ln \left(\frac{m T_{n}}{1-e^{-m T_{n}}}\right)\right) \\
& \triangleq H\left(T_{n}\right)
\end{aligned}
$$

Conversely, for the lower bound:

$$
\begin{aligned}
\max _{T}\left(\frac{1-e^{-m t_{0}^{*}}}{1-e^{-m T_{n+1}}} T_{n+1}-t_{0}^{*}\right) & =\max _{t_{0}^{*} \in\left[0, T_{n}\right]}\left(\frac{1-e^{-m t_{0}^{*}}}{1-e^{-m T_{n+1}}} T_{n+1}-t_{0}^{*}\right) \\
& =H\left(T_{n+1}\right)
\end{aligned}
$$

This maximum is reached for $T=\tilde{T} \in\left[T_{n+1}, T_{n}\right]$. Then:

$$
\begin{aligned}
\max _{T}\left(\frac{1-e^{-m t_{0}^{*}}}{1-e^{-m T}} T-t_{0}^{*}\right) & \geq\left(\frac{1-e^{-m t_{0}^{*}(\tilde{T})}}{1-e^{-m T}} \tilde{T}-t_{0}^{*}(\tilde{T})\right) \\
& \geq\left(\frac{1-e^{-m t_{0}^{*}(\tilde{T})}}{1-e^{-m T_{n+1}}} T_{n+1}-t_{0}^{*}(\tilde{T})\right) \\
& =H\left(T_{n+1}\right)
\end{aligned}
$$

We have shown that:

$$
H\left(T_{n+1}\right) \leq \max _{T \in\left[T_{n+1}, T_{n}\right]}\left(\frac{1-e^{-m t_{0}^{*}}}{1-e^{-m T}} T-t_{0}^{*}\right) \leq H\left(T_{n}\right)
$$

This, together with (14), implies that $\Delta \Pi_{n}$ is a decreasing function of $n$. Moreover $H(0)=0$ and $T_{n}$ tends to 0 as $n$ tends to infinity. Then $\Delta \Pi_{n}$ tends to 0 as $n$ tends to infinity. 


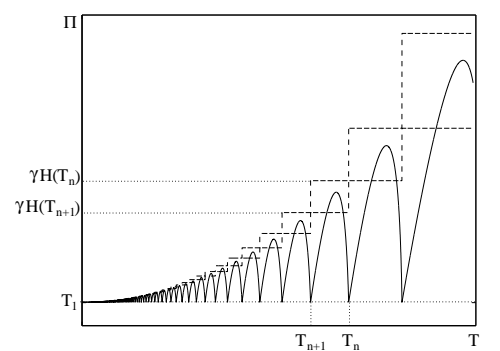

Fig. 1. Graphical illustration of Theorem 2: $\Pi$ as a function of the release period $T \in\left(0, T_{\min }\right)$ for some $n>n_{0}$ and with $\gamma=\mu g^{\prime}(0) /\left(\mu g^{\prime}(0)-m f^{\prime}(0)\right)$.

\subsection{Discussion}

Our main result directly comes from the interpretation of Theorem 2 . We have found the values $T_{n}$ of the release period $T$ that solve our min max problem through point (i-). However we use point (ii-) and a robustness argument to chose a particular $T_{n}$.

Indeed, we would like the optimality property to be robust to the level of invading pest population $x_{0}$ (that we do not know in advance), as well as to the model parameters. Our choice of $T=T_{n}$ will be made on the basis of the assumed nominal values of these parameters, but the uncertainties will make that our choice will not be a true $T_{n}$. Most probably our choice of $T$ will lie strictly between two true values of $T_{k}$ and then $\Pi$ would not be minimum. Therefore we should pick $T$ so that, in its neighborhood, $\Pi(T)$ does not attain too large values. We conclude from point (ii-) of Theorem 2 that we should then choose $T$ small (through $n$ large).

To summarize our results, provided the budget spent by time unit is large enough, prophylactic biological control is able to protect crops from invading pests. Moreover the most frequent releases of natural enemies minimize the worst case damage caused by pests on the crop, robustly to the initial pest population level and to model parameters.

\section{References}

1. D. Bainov. Impulsive Differential Equations. Longman, 1993.

2. C. Chicone. Ordinary Differential Equations with Applications. Texts in Applied Mathematics. Springer, 1999.

3. L.P. Jarvis. Managing Diseases in Greenhouse Crops. Amer. Phyto. Soc., 1992.

4. B. Liu, Y. Zhang, and L. Chen. The dynamical behaviors of a Lotka-Volterra predator-prey model concerning integrated pest management. Nonlinear Analysis: Real World Applications, 6:227-243, 2005.

5. B. Shulgin, L. Stone, and Z. Agur. Pulse vaccination stategy in the SIR endemic model. Bulletin of Mathematical Biology, 60:1123-1148, 1998.

6. V. M. Stern, R. F. Smith, R. van den Bosch, and K. S. Hagen. The integrated control concept. Hilgardia, 29:81-101, 1959. 